\title{
Clinical significance of the lymph node ratio in N1 breast cancer
}

\author{
Jaeho Kim, MD', Jin Hee Kim, MD, PhD'1, Ok Bae Kim, MD, PhD¹, Young Kee Oh, PhD', Seung Gyu Park, MD² \\ 'Department of Radiation Oncology, Keimyung University School of Medicine, Daegu; \\ ${ }^{2}$ Department of Radiation Oncology, Dogae Health Subcenter, Gumi, Korea
}

Purpose: The purpose of this study was to evaluate the prognostic value of the lymph node ratio (LNR), which was defined as the proportion of involved nodes of all dissected nodes, in pN1 breast cancer.

Materials and Methods: We retrospectively analyzed the clinical data of patients with pN1 breast cancer $(n=144)$ treated at Keimyung University Dongsan Medical Center, Daegu, Korea between 2001 and 2010. The median age was 46 years (range, 27 to 66 years). The LNR was $0.01-0.15$ (low LNR) in 130 patients and $>0.15$ (high LNR) in 14 patients. Sixty-five patients (45.1\%) had T1 tumors, $74(51.4 \%)$ had T2 tumors, and $5(3.5 \%)$ had T3 tumors. Eighty-eight patients $(61.1 \%)$ underwent total mastectomy and 56 (38.9\%) underwent partial mastectomy. Fifty-nine patients (41.0\%) underwent radiotherapy and 12 (8.3\%) underwent regional radiotherapy. The median follow-up period was 65 months.

Results: The 5- and 10-year disease-free survival (DFS) rates were $92.7 \%$ and $82.4 \%$, respectively. Univariate analyses revealed that high LNR $(p=0.004)$, total mastectomy $(p=0.006)$, no local radiotherapy $(p=0.036)$, and stage T2 or T3 $(p=0.010)$ were associated with worse DFS. In multivariable analysis, only high LNR $(p=0.015)$ was associated with worse DFS.

Conclusion: High LNR is an independent prognostic factor in $\mathrm{pN} 1$ breast cancer and could be an indication for adjuvant radiotherapy in these patients.

Keywords: Breast neoplasm, N1, Lymph node ratio

\section{Introduction}

Many randomized trials and a meta-analysis have shown that adequate postoperative locoregional radiotherapy for node-positive breast cancer reduces locoregional recurrence and improves the survival rate $[1,2]$. Therefore, the National Comprehensive Cancer Network guidelines state that locoregional radiation therapy should be considered for nodepositive patients after axillary lymph-node dissection [3]. There is also general consensus that locoregional radiotherapy should be considered for patients with four or more involved axillary nodes [4]. However, although the American Joint Committee on Cancer (AJCC) staging system reflects the disease state by emphasizing the prognostic importance of the absolute number of positive lymph nodes, there may be a discrepancy between the absolute number of positive nodes and the substantive extent of axillary-node metastasis, especially in patients with up to three positive nodes. Moreover, the

Received 04 January 2017, Revised 08 February 2017, Accepted 27 March 2017.

Correspondence: Jin Hee Kim, MD, PhD, Departments of Radiation Oncology, Keimyung University Dongsan Medical Center, Keimyung University School of Medicine, 56 Dalseong-ro, Jung-gu, Daegu 41931, Korea. Tel: +82-53-250-7665, Fax: + 82-53250-7984, E-mail: jhkim@dsmc.or.kr

(c) This is an Open Access article distributed under the terms of the Creative Commons Attribution Non-Commercial License (http://creativecommons.org/ licenses/by-nc/4.0/) which permits unrestricted non-commercial use, distribution, and reproduction in any medium, provided the original work is properly cited.

www.e-roj.org 
decision to administer regional radiotherapy based on the patient's pN1 node status differs between physicians [5-8].

In this context, the lymph node ratio (LNR), which is defined as the proportion of positive axillary lymph nodes of the total number of axillary lymph nodes removed, may resolve this problem [9-11]. Veronesi et al. [12] suggested that the LNR may reduce the discrepancy between clinical evaluation and the actual status of the lymph nodes that is due to the differing practices among physicians.

Therefore, recent studies have focused on the LNR in patients with pN1 breast cancer $[13,14]$. In this retrospective study, we assessed the prognostic value of the LNR and its potential use as an indication for locoregional radiotherapy after mastectomy in patients with pN1 breast cancer.

\section{Materials and Methods}

\section{Patients}

We performed a retrospective analysis of the clinical data of patients with pN1 invasive breast cancer who were treated at Keimyung University Dongsan Medical Center, Daegu, Korea between 2001 and 2010. The Institutional Review Board approval from Keimyung University Dongsan Medical Center on October 25, 2016. Eligible criteria were as follows: patients with unilateral breast cancer who underwent total mastectomy or partial mastectomy with whole breast radiotherapy, one to three positive lymph nodes, and no distant metastasis at the time of diagnosis. Patients who underwent neoadjuvant chemotherapy before surgery were excluded. In patients with a positive resection margin, re-excision was performed to achieve a margin-free status. In total, 149 patients were initially screened for this study, of which 5 patients underwent sentinel lymph node biopsy and were excluded from the study. Therefore, 144 patients were included in this study. All patients received postoperative adjuvant chemotherapy, with an adriamycin/cyclophosphamide-paclitaxel regimen in most patients $(139,93.3 \%)$. All of the patients underwent either total mastectomy or partial mastectomy with axillary lymph node dissection. Lymph node dissection was usually performed up to level II. None of the patients had any serious comorbidity.

The local radiotherapy field consisted of the whole breast in partial mastectomy patients or the chest wall in total mastectomy patients. All of the partial mastectomy patients underwent local radiotherapy. Of the total mastectomy patients, only three underwent local radiotherapy and all of them underwent regional radiotherapy. The breast or chest wall was irradiated with $6 \mathrm{MV}$ photons; the median dose of local radiotherapy was 5,040 cGy (range, 4,500 to 5,400 cGy), with $180 \mathrm{cGy}$ per fraction. An electron boost was applied to the tumor bed in all partial mastectomy patients after local radiotherapy (median, 1,000 cGy/5 fractions). The regional radiotherapy field included the supraclavicular axillary lymph nodes. The internal mammary lymph nodes (IMN) were also included in patients with poor prognostic factors such as primary tumor located in an inner quadrant, large tumor, or lymphovascular invasion. Among patients who underwent regional radiotherapy, four patients received radiotherapy with an IMN field. The dose of regional radiation therapy was 5,000 $c G y$, with 200 cGy given per fraction. The median duration of radiotherapy was 44 days.

\section{Clinical and pathological factors and lymph node status}

Age, tumor size, operation type, local radiotherapy, regional lymph node radiotherapy, adjuvant hormone therapy, pathology, resection margin, Iymphovascular invasion, extracapsular extension, molecular subtype, histological grade, and LNR were used to assess the risk of recurrence. Staging was based on the 7th edition of the AJCC Cancer Staging Manual. A close margin was defined as the presence of invasive carcinoma within $2 \mathrm{~mm}$ of the surgical resection margin. Luminal type $A$ was defined as hormone receptorpositive, HER2-negative and Ki67 <14\%. Luminal type B was defined as hormone receptor-positive plus either Ki67 $\geq 14 \%$ or HER2-positive. HER2-enriched was defined as hormone receptor-negative and HER2-positive. Triple negative was defined as hormone receptor-negative and HER2-negative.

The LNR was arbitrarily set to 0.01 units and the logrank test was performed with disease-free survival (DFS) to determine the appropriate LNR cutoff value. Based on the results of these analyses, we used a cutoff value of 0.15 , which yielded the most significant result and divided patients into two groups according to the LNR, low LNR $(\leq 0.15)$ and high LNR (>0.15).

\section{Follow-up and endpoints}

DFS was defined as the time from the date of diagnosis to locoregional recurrence, distant metastasis, or contralateral invasive breast cancer. The patients were followed-up every 3-6 months after surgery with history and physical examinations. Mammography was performed every 12 months. Additional imaging studies were performed in patients with suspicious clinical signs or symptoms. 
Table 1. Patient characteristics

\begin{tabular}{|c|c|}
\hline Characteristic & Value \\
\hline Age (yr) & $46(27-66)$ \\
\hline$<40$ & $8(5.6)$ \\
\hline$\geq 40$ & $136(94.4)$ \\
\hline \multicolumn{2}{|l|}{ Tumor size } \\
\hline $\mathrm{T} 1$ & $65(45.1)$ \\
\hline $\mathrm{T} 2$ & $74(51.4)$ \\
\hline T3 & $5(3.5)$ \\
\hline \multicolumn{2}{|l|}{ Number of positive lymph nodes } \\
\hline 1 & $80(55.6)$ \\
\hline 2 & $46(31.9)$ \\
\hline 3 & $18(12.5)$ \\
\hline Number of dissected lymph nodes & $17(5-43)$ \\
\hline$\leq 20$ & $85(59.0)$ \\
\hline$>20$ & $59(41.0)$ \\
\hline LNR & $0.09(0.02-0.23)$ \\
\hline $\operatorname{Low}(\leq 0.15)$ & $130(90.3)$ \\
\hline $\operatorname{High}(>0.15)$ & $14(9.7)$ \\
\hline \multicolumn{2}{|l|}{ Operation type } \\
\hline Total mastectomy & $88(61.1)$ \\
\hline Partial mastectomy & $56(38.9)$ \\
\hline \multicolumn{2}{|l|}{ Local radiotherapy } \\
\hline No & $85(59.0)$ \\
\hline Yes & $59(41.0)$ \\
\hline \multicolumn{2}{|l|}{ Regional radiotherapy } \\
\hline No & $132(91.7)$ \\
\hline Yes & $12(8.3)$ \\
\hline \multicolumn{2}{|l|}{ Pathology } \\
\hline IDC & $139(96.5)$ \\
\hline Other & $5(3.5)$ \\
\hline \multicolumn{2}{|l|}{ Histologic grade } \\
\hline$|\&| \mid$ & $62(43.1)$ \\
\hline III & $72(50.0)$ \\
\hline Unknown & $10(6.9)$ \\
\hline \multicolumn{2}{|l|}{ LVI } \\
\hline No & 57 (39.6) \\
\hline Yes & $74(51.4)$ \\
\hline Unknown & $13(9.0)$ \\
\hline \multicolumn{2}{|l|}{ ECE } \\
\hline No & $100(69.4)$ \\
\hline Yes & $21(14.6)$ \\
\hline Unknown & $23(16.0)$ \\
\hline \multicolumn{2}{|l|}{ Resection margin (mm) } \\
\hline Close $(<2)$ & $21(14.6)$ \\
\hline Clear $(\geq 2)$ & $122(84.7)$ \\
\hline Unknown & $1(0.7)$ \\
\hline \multicolumn{2}{|l|}{ Molecular subtype } \\
\hline Luminal $A^{\text {a) }}$ & $63(43.8)$ \\
\hline Luminal $B^{\text {b) }}$ & $41(28.5)$ \\
\hline HER2-enriched ${ }^{c)}$ & $7(4.9)$ \\
\hline Triple negative $^{d)}$ & $22(15.3)$ \\
\hline Unknown & $11(7.6)$ \\
\hline \multicolumn{2}{|l|}{ Adjuvant hormone therapy } \\
\hline No & $31(21.5)$ \\
\hline Yes & $113(78.5)$ \\
\hline
\end{tabular}

Values are presented as median (range) or number (\%). LNR, lymph node ratio; IDC, invasive ductal carcinoma; LVI, Iymphovascular invasion; ECE, extracapsular extension.

a) Hormone receptor-positive, HER2-negative and Ki67 <14\%.

b) Hormone receptor-positive \& Ki67 $\geq 14 \%$ or HER2-positive.

c) Hormone receptor-negative and HER2-positive.

d) Hormone receptor-negative and HER2-negative.
Table 2. Clinical status and patterns of failure

\begin{tabular}{lc}
\hline & Value \\
\hline Follow-up (mo) & $65(7-165)$ \\
Clinical status & $125(86.8)$ \\
NED & $6(4.2)$ \\
Alive with disease & $13(9.0)$ \\
Cause-specific death & $0(0)$ \\
Intercurrent death & \\
Pattern of failure & $3(15.8)$ \\
LR only & $1(5.3)$ \\
RR only & $2(10.5)$ \\
RR + DM & $13(68.4)$ \\
DM only &
\end{tabular}

Values are presented as median (range) or number (\%).

$N E D$, no evidence of disease; $L R$, local recurrence; $R R$, regional recurrence; DM, distant metastasis.

\section{Statistical analysis}

Data were analyzed with SPSS ver. 18.0 for Windows (SPSS Inc., Chicago, IL, USA). The Kaplan-Meier method was used to analyze DFS and statistical significance was determined using log-rank tests. Cox stepwise regression analysis was used for multivariable analysis. Statistically significant variables in the univariate analysis $(p<0.05)$ were included in the Cox regression model. Statistical significance was set at $p<0.05$.

\section{Results}

The characteristics of the 144 patients with $\mathrm{pN} 1$ breast cancer are summarized in Table 1. Of 144 evaluable patients, 65 (45.1\%), $74(51.4 \%)$, and 5 (3.5\%) had T1, T2, and T3 lesions, respectively. More than 20 lymph nodes were dissected in $59(41.0 \%)$ patients. Based on the LNR cutoff value of 0.15 , the low LNR group comprised 130 (90.3\%) patients and the high LNR group comprised 14 (9.7\%) patients. The LNR was statistically independent of other prognostic factors such as age, operation type, T stage, resection margin, lymphovascular invasion, molecular subtype, and histologic grade.

The median follow-up time was 65 months (range, 7 to 165 months). The 5- and 10-year DFS rates were $92.7 \%$ and $82.4 \%$, respectively (Fig. 1). The patterns of failure are listed in Table 2. When all 19 recurrences were classified according to regional radiotherapy, recurrence was noted in $18(13.6 \%)$ patients who did not undergo regional radiotherapy while only $1(8.3 \%)$ patient in the regional radiotherapy group suffered distant metastasis. None of the patients in the regional radiotherapy group experienced locoregional recurrence. There were no cases of contralateral breast cancer recurrence. None 
of the patients experienced adverse effects associated with radiotherapy.

In univariate analyses, large tumor, high LNR, absence of local radiotherapy, and operation type (total mastectomy) were associated with poor DFS. In the subsequent multivariable analysis, only high LNR was significantly associated with poor DFS (Tables 3, 4).

To further evaluate the prognostic role of $L N R$, we

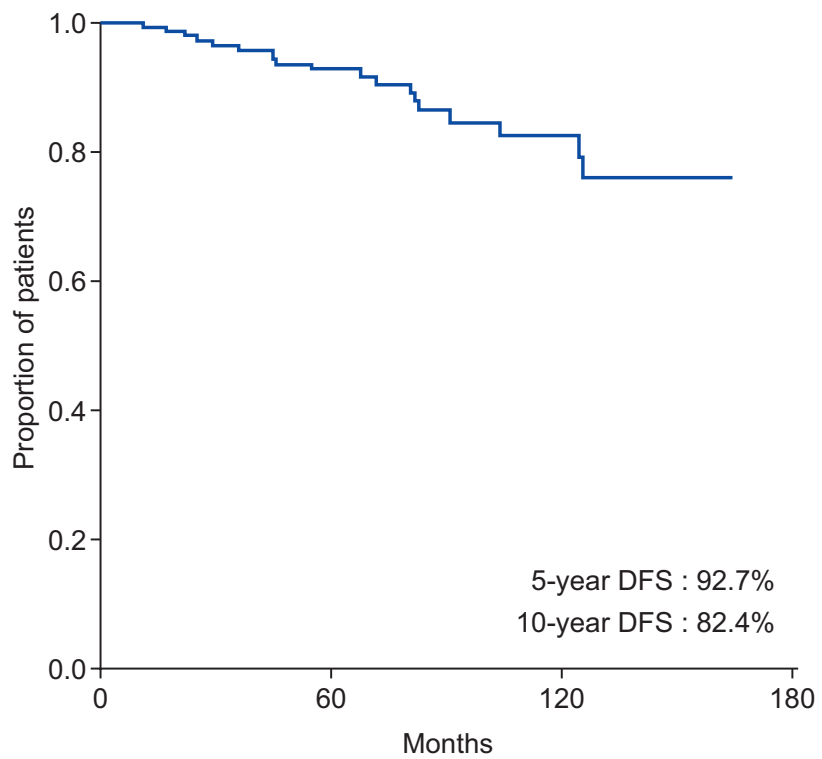

Fig. 1. Kaplan-Meier estimates of disease-free survival (DFS).

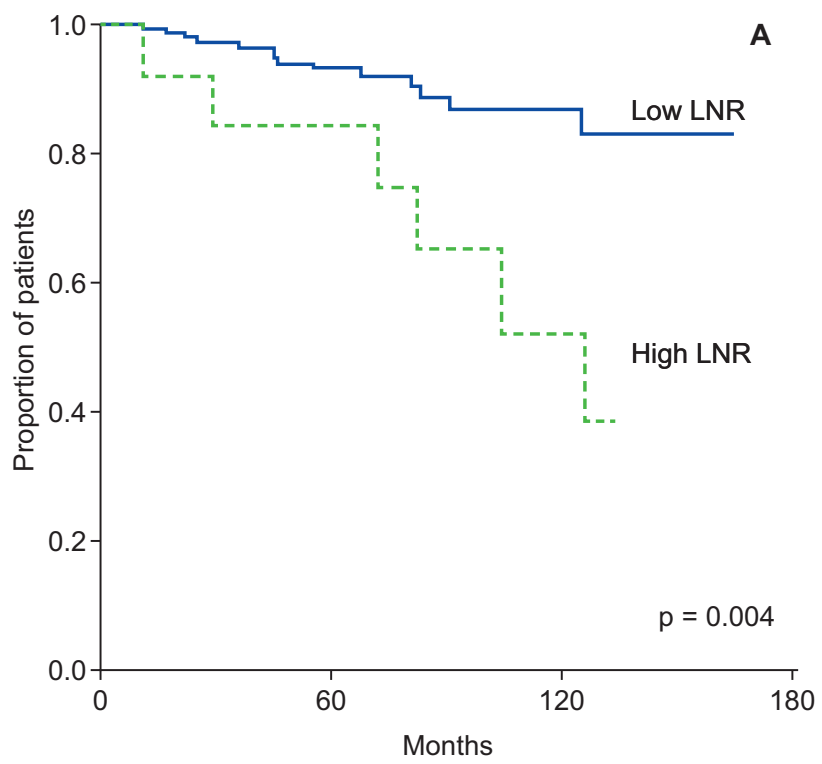

analyzed DFS in 132 patients who did not undergo regiona radiotherapy, of which 13 (9.8\%) were in the high LNR group. DFS was significantly worse in the high LNR group than in the low LNR group (Fig. 2).

\section{Discussion and Conclusion}

It is widely accepted that postoperative locoregional radiation therapy reduces locoregional recurrence and mortality in patients with lymph-node-positive breast cancer [1]. A metaanalysis conducted by the Early Breast Cancer Trialists' Collaborative Group (EBCTCG) and a report of the MA.20 study indicate that regional radiotherapy significantly reduced the overall recurrence rate in patients with lymph-node-positive breast cancer, even when adjuvant systemic chemotherapy was administered $[15,16]$. The EBCTCG study also showed that the absolute benefit of radiotherapy for $\mathrm{N} 1$ breast cancer patients is small, but more effective radiotherapeutic regimens introduced since that study should provide better outcomes. Regional radiotherapy may have considerable benefits in selected patients with $\mathrm{N} 1$ breast cancer. However, the prognostic factors associated with reduced risk of locoregional recurrence after regional radiotherapy have not been reliably defined when dividing patients into subgroups. Therefore, there are currently no prognostic factors that provide clear support for locoregional radiotherapy in patients with $\mathrm{pN} 1$ breast cancer.

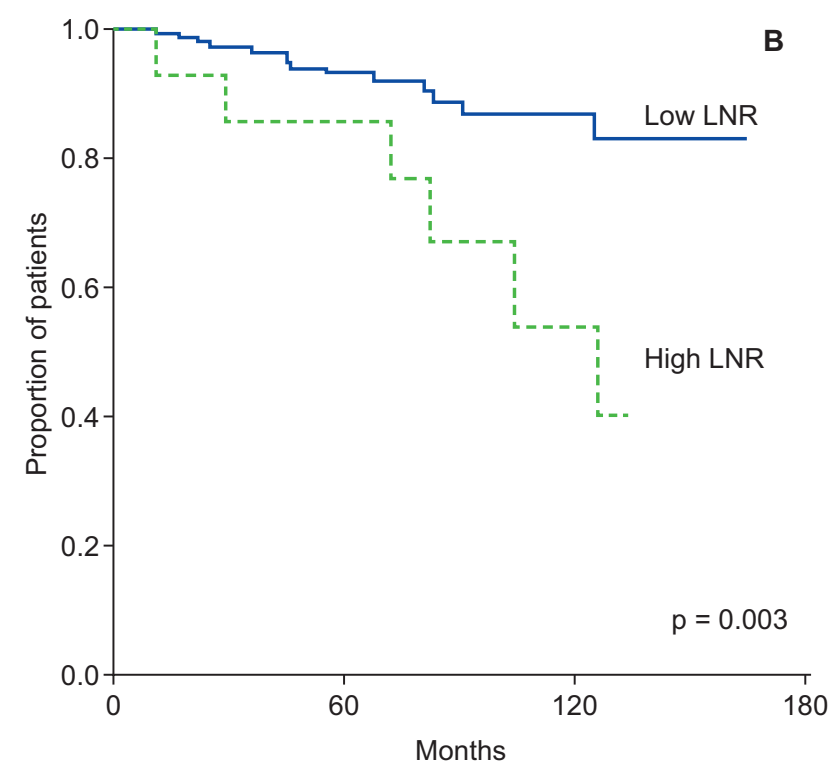

Fig. 2. Comparison of disease-free survival between patients with a low lymph node ratio (low LNR, solid line) and patients with a high lymph node ratio (high LNR, dashed line). (A) All patients. (B) Patients who did not undergo regional radiotherapy. 
As described above, the value of LNR as a prognostic factor has been examined in multiple studies. Several studies suggested that the LNR status is a better predictor of prognosis compared with the nodal status recommended by

Table 3. Univariate analysis for disease-free survival

\begin{tabular}{|c|c|c|}
\hline & $5-y r$ DFS $(\%)$ & p-value \\
\hline Age (yr) & & 0.124 \\
\hline$<40$ & 87.5 & \\
\hline$\geq 40$ & 93.1 & \\
\hline Tumor size & & 0.010 \\
\hline $\mathrm{T} 1$ & 96.7 & \\
\hline T2 \& T3 & 89.5 & \\
\hline LNR group & & 0.004 \\
\hline Low $(\leq 0.15)$ & 93.5 & \\
\hline High (>0.15) & 85.7 & \\
\hline Operation type & & 0.006 \\
\hline Total mastectomy & 89.1 & \\
\hline Partial mastectomy & 98.0 & \\
\hline Local radiotherapy & & 0.036 \\
\hline No & 90.7 & \\
\hline Yes & 96.0 & \\
\hline Regional radiotherapy & & 0.900 \\
\hline No & 93.0 & \\
\hline Yes & 83.3 & \\
\hline Histologic grade & & 0.365 \\
\hline$|\&| \mid$ & 93.3 & \\
\hline III & 92.7 & \\
\hline LVI & & 0.961 \\
\hline No & 91.1 & \\
\hline Yes & 92.8 & \\
\hline ECE & & 0.908 \\
\hline No & 92.6 & \\
\hline Yes & 94.7 & \\
\hline Resection margin (mm) & & 0.702 \\
\hline Close $(<2)$ & 84.3 & \\
\hline Clear $(\geq 2)$ & 94.1 & \\
\hline Molecular subtype & & 0.843 \\
\hline Hormone receptor (+) & 93.0 & \\
\hline Hormone receptor (-) & 88.9 & \\
\hline Adjuvant hormone therapy & & 0.615 \\
\hline No & 85.9 & \\
\hline Yes & 94.5 & \\
\hline
\end{tabular}

DFS, disease-free survival; LNR, lymph node ratio; LVI, lymphovascular invasion; ECE, extracapsular extension.

Table 4. Multivariable analysis for disease-free survival

\begin{tabular}{lcc}
\hline & $H R(95 \% \mathrm{Cl})$ & $\mathrm{p}$-value \\
\hline $\begin{array}{c}\text { Operation type } \\
\text { (total vs. partial mastectomy) }\end{array}$ & $0.147(0.014-1.581)$ & 0.114 \\
$\begin{array}{l}\text { Local radiotherapy } \\
\text { (no vs. yes) }\end{array}$ & $1.652(0.230-11.875)$ & 0.618 \\
T stage (T1 vs. T2/T3) & $2.444(0.667-8.953)$ & 0.177 \\
LNR (low vs. high) & $3.453(1.273-9.361)$ & 0.015 \\
\hline
\end{tabular}

$\mathrm{HR}$, hazard ratio; $\mathrm{Cl}$, confidence interval; LNR, lymph node ratio. the AJCC, especially when estimating the benefit of additional radiotherapy after surgery $[7-9,12,13]$.

No consensus has been reached on the appropriate LNR cutoff value. The cutoff value for low-risk LNR is often set within a range of about $0.20-0.25$. In our study, we used a cutoff value of 0.15 , which is fairly low compared with the values used in other studies $[8,9,14,15]$. However, our study only included patients with $\mathrm{pN} 1$ breast cancer. Therefore, most of the patients had low LNRs, and a cutoff value of 0.20 was deemed unsuitable in this single-institution study with a small sample size. The LNR is strongly influenced by the total number of lymph nodes dissected. In our study, the median number of dissected lymph nodes was 17, which is higher than the numbers in earlier studies $[13,16]$. For these reasons, the LNR was relatively low in this study. We suggest that further studies are required to determine the most appropriate cutoff value.

This study had some limitations. First, it was a retrospective study of a single institution, so it is difficult to extrapolate the conclusions of the study to the general population. For this reason, some prognostic factors that were significant in other larger studies showed borderline significance or were not significant in our study. Second, it is difficult to define the obvious reasons for performing locoregional radiotherapy in total mastectomy patients and the obvious criteria for IMN irradiation. Third, the radiotherapeutic regimens were unclear in some patients; for example, it is unclear why one partial mastectomy patient underwent radiotherapy with 4,500 cGy. Finally, we cannot confirm the clinical value of the LNR based on our findings because our study was not sufficiently powered. Nevertheless, the LNR may be useful to estimate the clinical value of radiotherapy, although a large multiinstitutional study is necessary to confirm this possibility.

Although locoregional radiation therapy reduces the likelihood of locoregional recurrence, it can cause arm edema by destroying the lymphatic drainage system already damaged by surgery and brachial plexopathy. Therefore, locoregional radiotherapy may be unsuitable for some patients, although the number of such patients is expected to be very small. In this context, the LNR may provide a more reliable indication for locoregional radiation therapy than nodal status alone. In this study, no patient showed severe complications, but because the study was retrospective, it was not possible to calculate the patients' complication scores. Prospective randomized trials should provide additional information regarding the possibility of complications in this context.

In conclusion, our results suggest that a high LNR is an 
independent prognostic factor in patients with $\mathrm{pN} 1$ breast cancer, and could be an indication for adjuvant regional radiotherapy in these patients. However, a multicenter study is needed to verify the value of LNR as an indication for adjuvant regional radiotherapy in patients with $\mathrm{pN} 1$ breast cancer.

\section{Conflict of Interest}

No potential conflict of interest relevant to this article was reported.

\section{References}

1. Clarke $M$, Collins $R$, Darby $S$, et al. Effects of radiotherapy and of differences in the extent of surgery for early breast cancer on local recurrence and 15-year survival: an overview of the randomised trials. Lancet 2005;366:2087-106.

2. Overgaard M, Hansen PS, Overgaard J, et al. Postoperative radiotherapy in high-risk premenopausal women with breast cancer who receive adjuvant chemotherapy. Danish Breast Cancer Cooperative Group 82b Trial. N Engl J Med 1997;337:949-55

3. National Comprehensive Cancer Network. NCCN Clinical Practice Guidelines in Oncology: breast cancer (version 2.2016) [Internet]. Fort Washington, PA: National Comprehensive Cancer Network; c2017 [cited 2017 Jun 1]. Available from: https://www.nccn.org/professionals/physician_gls/f_ guidelines.asp.

4. Overgaard M, Nielsen HM, Overgaard J. Is the benefit of postmastectomy irradiation limited to patients with four or more positive nodes, as recommended in international consensus reports? A subgroup analysis of the DBCG 82 betc randomized trials. Radiother Oncol 2007;82:247-53.

5. Han TJ, Kang EY, Jeon W, et al. The prognostic value of the nodal ratio in N1 breast cancer. Radiat Oncol 2011;6:131.

6. Recht A, Bartelink H, Fourquet A, et al. Postmastectomy radiotherapy: questions for the twenty-first century. J Clin Oncol 1998;16:2886-9.

7. Recht $A$, Edge SB, Solin $\sqcup$, et al. Postmastectomy radiotherapy: clinical practice guidelines of the American Society of Clinical
Oncology. J Clin Oncol 2001;19:1539-69.

8. Truong PT, Olivotto IA, Whelan TJ, Levine $M$; Steering Committee on Clinical Practice Guidelines for the Care and Treatment of Breast Cancer. Clinical practice guidelines for the care and treatment of breast cancer: 16. Locoregional postmastectomy radiotherapy. CMAJ 2004;170:1263-73.

9. Megale Costa $\sqcup$, Soares HP, Gaspar HA, et al. Ratio between positive lymph nodes and total dissected axillaries lymph nodes as an independent prognostic factor for diseasefree survival in patients with breast cancer. Am J Clin Oncol 2004;27:304-6.

10. Vinh-Hung V, Verkooijen HM, Fioretta G, et al. Lymph node ratio as an alternative to $\mathrm{pN}$ staging in node-positive breast cancer. J Clin Oncol 2009;27:1062-8.

11. Woodward WA, Vinh-Hung V, Ueno NT, et al. Prognostic value of nodal ratios in node-positive breast cancer. J Clin Oncol 2006;24:2910-6.

12. Veronesi U, Zurrida S, Viale G, Galimberti V, Arnone P, Nole F. Rethinking TNM: a breast cancer classification to guide to treatment and facilitate research. Breast J 2009;15:291-5.

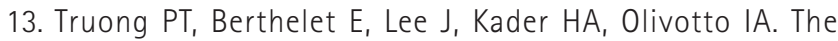
prognostic significance of the percentage of positive/dissected axillary lymph nodes in breast cancer recurrence and survival in patients with one to three positive axillary lymph nodes. Cancer 2005;103:2006-14.

14. Truong PT, Woodward WA, Thames HD, Ragaz J, Olivotto IA, Buchholz TA. The ratio of positive to excised nodes identifies high-risk subsets and reduces inter-institutional differences in locoregional recurrence risk estimates in breast cancer patients with 1-3 positive nodes: an analysis of prospective data from British Columbia and the M. D. Anderson Cancer Center. Int J Radiat Oncol Biol Phys 2007;68:59-65.

15. EBCTCG (Early Breast Cancer Trialists' Collaborative Group). Effect of radiotherapy after mastectomy and axillary surgery on 10-year recurrence and 20-year breast cancer mortality: meta-analysis of individual patient data for 8135 women in 22 randomised trials. Lancet 2014;383:2127-35.

16. Whelan TJ, Olivotto IA, Levine MN. Regional nodal irradiation in early-stage breast cancer. N Engl J Med 2015;373:1878-9. 